E-mail:xbywb@ouc.edu.cn

\title{
Erratum to: Quorum-Sensing of Bacteria and Its Application
}

(C) Ocean University of China and Science Press and Springer-Verlag Berlin Heidelberg 2010

Retraction: Jiang Guoliang and Su Mingxia. Quorum-Sensing of Bacteria and Its Application. Journal of Ocean University of China 2009, 8 (4): 385-391. DOI: 10.1007/s11802-009-0385-1

This article is retracted because of copyright violation.

Journal of Ocean University of China July 19, 2010 